\title{
Szenarien zur Transformation von Energieversorgungs- systemen als Voraussetzung für die Ableitung von Anforderungen an zukünftige Kompetenzen
}

\author{
Frank Hartmann, Dana Mietzner*
}

\section{Zusammenfassung}

Die Transformation von Energieversorgungssystemen ist durch ein hohes Maß an Unsicherheiten gekennzeichnet. Dies trifft auch auf die Frage zu, welche Qualifikationen und Kompetenzen in der Zukunft notwendig sind, um im Transformationsprozess passfähig zu sein. Eine Möglichkeit des Umgangs mit diesen Unsicherheiten besteht in der systematischen Herausarbeitung von Anforderungen an Qualifikationen und Kompetenzen, die sich auf die Antizipation von möglichen Zukünften der Energieversorgung stützt. Aus diesem Grund gehen die Autoren in ihrem Beitrag der Frage nach, wie diese Anforderungen an Qualifikationen und Kompetenzen bei der Transformation von Energieversorgungssystemen systematisch ermittelt werden können. Hierbei greifen sie auf den Transformationsansatz bzw. die Multi Level Perspective (englisch) zurück und entwickeln ihren Ansatz für ein spezifisches Szenarioanalyse-Design. Im Anschluss daran werden Anforderungen an Qualifikationen und Kompetenzen vor dem Hintergrund unterschiedlicher Szenarien abgeleitet.

\section{Abstract}

The transformation of energy supply systems is characterized by a high degree of uncertainty. This also applies to the question, which skills and competencies are needed in the future in order to be suitable in the transformation process. One way of dealing with these uncertainties is the systematic elaboration of requirements for skills and competences, which is based on the anticipation of possible futures of the energy supply. Thus, the authors discuss how such requirements for qualifications and skills can be systematically identified. The authors use the transformation approach, respectively the multi-level perspective, and, on this basis, develop an approach for the specific design of a scenario analysis. Subsequently, requirements for qualifications and competences are derived.

\section{Ausgangssituation}

Die begrenzte Verfügbarkeit fossiler Rohstoffe, die mit deren Nutzung verbundenen negativen Folgen für das Klima sowie der Atomausstieg sind wesentliche Treiber für die Transformation von Energieversorgungssystemen. Die damit im Zusammenhang stehenden Herausforderungen haben auch eine regionale Dimension. Regionen verfügen über unterschiedliche Bedingungen und Voraussetzungen, um den Umbau ihrer Energieversorgungssysteme voranzutreiben. Hierzu gehören beispielsweise ihre Ausstattung mit natürlichen Ressourcen, die ökonomische Leistungsfähigkeit und Ausgestaltung ihres politischen Systems oder auch die verschiedenen institutionellen Arrangements. Seit Jahrzehnten sind damit verbundene Fragestellungen Gegenstand von Forschung, öffentlichen Debatten und politischen Entscheidungsprozessen (vgl. z. B. Gailing et al. 2013, WBGU 2011a, Fournier et al. 2013). Die Komplexität der Thematik hat zu einer kaum mehr übersehbaren Vielzahl von Ansätzen, Konzepten, Vorgehensweisen und Strategien für den Umgang mit der Energieversorgungsproblematik geführt. Auch innerhalb von Ländern und Regionen existieren unterschiedliche Sicht- und Herangehensweisen. Es werden Auseinander- setzungen um die richtige Strategie geführt, basierend auf unterschiedlichen Interessenlagen und vor dem Hintergrund unterschiedlicher Stellungen und Funktionen von Akteuren in der Gesellschaft. Beispiele hierfür sind die Debatten über eine Klimaabgabe für ältere Kohlekraftwerke oder über die Sicherstellung der Beteiligung von Kernkraftwerksbetreibern an den Rückbaukosten für Kernkraftwerke.

Dies zeigt, dass der Umbau eines Energieversorgungssystems durch ein hohes Maß an Unsicherheiten gekennzeichnet ist. Gleiches gilt für die Frage, welche Qualifikationen und Kompetenzen zukünftig erforderlich sind vor dem Hintergrund der Transformation 
des Energieversorgungssystems.

Eine Möglichkeit des Umgangs mit diesen Unsicherheiten besteht in der systematischen Herausarbeitung von Anforderungen an Qualifikationen und Kompetenzen, die sich auf die Antizipation von möglichen Zukünften der Energieversorgung stützt. Die Analyse bestehender Ausund Weiterbildungsangebote oder die Orientierung an gegenwärtigen Nachfragen der Unternehmen reicht hierfür nicht aus. Aus diesem Grund gehen die Autoren in diesem Beitrag der Frage nach, wie zukünftige Anforderungen an Qualifikationen und Kompetenzen bei der Transformation von Energieversorgungssystemen systematisch ermittelt werden können.

Der vorliegende Beitrag beginnt mit der konzeptionellen Einbettung der Fragestellung und fokussiert hierbei auf Transformationsansätze und das Verständnis von Energieversorgungssystemen. Im Anschluss daran wird ein Szenarioanalyseprozess unter methodischen Aspekten skizziert. Hieran anknüpfend werden Anforderungen an neue Qualifikationen und Kompetenzen abgeleitet.

\section{Konzeptioneller Hintergrund}

Um einen geeigneten Zugang zur Problematik des Umbaus von Energieversorgungssystemen zu entwickeln, ist ein konzeptioneller Rahmen erforderlich, der es ermöglicht, den Untersuchungsgegenstand in geeigneter Form zu beschreiben und seine Entwicklung in übergeordnete gesellschaftliche Kontexte einzuordnen. Hierfür ist der Transformationsansatz geeignet, der in verschiedenen Facetten und mit unterschiedlichen Schwerpunkten seit vielen Jahren thematisiert wird (vgl. Grin et al. 2010, WBGU 2011b). In einem sehr umfassenden Verständnis und fokussiert auf Umwelt und Klima wird er beispielsweise im Hauptgutachten des wissenschaftlichen Beirates der Bundesregierung „Globale Umweltveränderungen“ unter dem Titel „Welt im Wandel - Gesellschaftsvertrag für eine große Transformation“ aus dem Jahr 2011 entwickelt (vgl. WBGU 2011b). Gesellschaftliche Transformationen entstehen - entsprechend diesem Verständnis - durch aufeinander bezoge- ne Veränderungen von Technologien, gesellschaftlichen Institutionen und individuellen Verhaltensweisen in gesellschaftlichen Subsystemen (WBGU 2011b: 342). Um sie verstehen zu können, ist es erforderlich, eine Systematik zu entwickeln, die es erlaubt, Prozesse in ihrer Dynamik zu beschreiben, Treiber von Veränderungen herauszuarbeiten sowie damit im Zusammenhang stehende Akteurskonstellationen sowie Handlungsebenen sichtbar zu machen (WBGU 2011b: 87). Geeignet hierfür ist die von Geels und Schot im Jahr 2007 vorgeschlagene multilevelperspective für Transformationsprozesse, die 2010 von Grin (Grin et al. 2010) aufgegriffen wurde, um zugängliche Handlungsebenen im Transformationsprozess zu bestimmen. Das Ziel dieses Ansatzes besteht darin, Komplexitäten, Vielschichtigkeiten und Ungleichzeitigkeiten der Transformationsprozesse zu reflektieren und gleichzeitig ein Modell verfügbar zu haben, das radikal vereinfacht.

Dieses Modell ist aus der Sicht der Autoren auch geeignet, um den Kontext für den Umbau von Energiesystemen zu strukturieren. Im Zentrum des Modells stehen drei unterschiedliche Handlungsebenen, die sich wechselseitig bedingen und dynamisch sind (Geels 2007, Geels \& Schot 2007). Durch Veränderungen und Dynamiken in diesen Handlungsebenen und deren Interaktion entstehen nach Auffassung dieser Autoren Möglichkeitsräume für Transformation. Hierbei handelt es sich um die Ebene "Sociotechnical landscape“ als exogener Makrokontext, die Ebene "Socio-technical regime" als Transformationsgegenstand im engeren Sinne sowie die Ebene "Niche innovations" als Ebene, in der das Innovationsgeschehen auf der Mikroebene als ein wesentlicher Treiber für die Transformation des soziotechnischen Regimes wirkt. Das etablierte soziotechnische Regime, als System von Technologien, Märkten, Industrien, Wissenschaftssystemen und Kulturen, entwickelt sich unter dem Einfluss der soziotechnischen Landschaft, die Druck auf das Regime sowohl in Richtung von Veränderung als auch möglicherweise in Richtung von Konservierung ausübt. Die soziotechnische Landschaft ist durch lange Zyklen und Trends charakterisiert, die nicht ohne weiteres durch Akteurskonstellationen beeinflussbar sind. Die Ebene der Nischeninnovationen beschreibt auf der Mikroebene die Entstehung radikaler Innovationen, die in bestimmten Konstellationen die Chance haben, merklichen Einfluss auf das soziotechnische Regime zu nehmen. Insbesondere die Destabilisierung des etablierten soziotechnischen Regimes eröffnet windows of opportunity für radikale Nischeninnovationen. Eine Gleichrichtung der Prozesse auf den drei Ebenen ermöglicht Innovationen mit Durchbruchcharakter, die beginnen, vorhandene Märkte zu dominieren und mit dem bestehenden Regime zu konkurrieren (Geels 2007: 400). Das hier skizzierte Modell kann sowohl für die Beschreibung einer oben angesprochenen großen gesellschaftlichen Transformation herangezogen werden als auch für die Analyse der Transformation nationaler Energieversorgungssysteme.

Während es zahlreiche Hinweise dafür gibt, dass sich die soziotechnische Landschaft im Zusammenhang mit der Transformation von Energieversorgungssystemen global entwickelt, scheint die Übertragung dieser Annahme auf ein soziotechnisches Regime problematisch. Dafür sind die Entwicklungen weltweit historisch gesehen viel zu unterschiedlich verlaufen und die Ausprägung der soziotechnischen Regimes der Energieversorgung ist sowohl in struktureller Hinsicht (z. B. Energiemix) als auch in akteursbezogener Hinsicht (z. B. Regulierung) viel zu differenziert. Insofern rücken auch stärker räumliche Aspekte im Zusammenhang mit der Transformation von soziotechnischen Regimes in das Blickfeld, die bisher weitgehend vernachlässigt wurden (vgl. Truffer 2013). Truffer hat diese Fragestellung explizit aufgegriffen und untersucht, welche Rolle lokale Formierungsprozesse in der Transformation beispielsweise von Energieversorgungssystemen spielen. Er hat zu Recht das Problem in den Fokus genommen, wie lokale, nationale und internationale Akteure, Institutionen und Prozesse aufeinander bezogen werden können. In eine ähnliche Richtung gehen Argumentationen von Ohlhorst et al., die jedoch eher auf die Koordination von Aktivitäten auf der subnationalen Ebene 
fokussieren (Ohlhorst et al. 2013). Für die Konzipierung der im Mittelpunkt dieses Beitrages stehenden Analyse wurden die wichtigsten Systemzusammenhänge der Energieversorgung, verstanden als soziotechnisches Regime, auf der nationalen Ebene verortet, auch wenn zahlreiche Dezentralisierungsaktivitäten auf regionaler Ebene an Bedeutung gewonnen haben und internationale Entwicklungen nationale Strategien in zunehmendem Maße beeinflussen.

Was die Nischenebene des Modells betrifft, so gehen die Autoren davon aus, dass Innovationen unterschiedlichsten Typs (Produkt- und Prozessinnovationen, soziale Innovationen wie Geschäftsmodellinnovationen, neue Organisations- und Beteiligungsmodelle oder auch neue Institutionen) mit verschiedenen Neuheitsgraden auf allen Ebenen, von lokal über regional und national bis global vorangebracht werden und auf das nationale soziotechnische Regime wirken.

Wird die Transformation des deutschen Energieversorgungssystems rückwirkend betrachtet, ist erkennbar, dass dieser Prozess bereits in den 1970er Jahren mit der Bewegung der Kernenergiegegner begann. Sie wurde auch durch Vorreiter einer auf Ressourcenschonung bedachten globalen ökonomischen Sichtweise gespeist, die sich beispielsweise im Bericht „Die Grenzen des Wachstums" des Club of Rome aus dem Jahr 1972 zeigte (Meadows et al. 1972). Bestätigt durch die Schocks, welche die Erdölkrisen der Jahre 1973 und 1979/80 auslösten, formierte sich in Deutschland in den 70er und 80er Jahren eine starke Umweltschutzbewegung, etwa im Kampf gegen das Waldsterben und insbesondere gegen die Kernenergienutzung. Diese Umweltbewegung erhielt weiteren Auftrieb und eine breitere Basis im Zuge der Kernenergiekatastrophe in Tschernobyl im Jahr 1986 (vgl. Jacobsson \& Lauber 2006). Diese Entwicklungen können als Ausdruck von Veränderungen in der sozioökonomischen Landschaft bzw. als Reaktionen auf diese Veränderungen interpretiert werden. Nach Jacobsen und Lauber führte das zu einer formativen Periode für den Übergang zu einem neuen System der Energieversorgung. Diese formative Periode hat nach Jacobsson und Lauber vier wichtige Merkmale (Jacobsson \& Lauber 2006): Sie ist (1) durch einen institutionellen Wandel gekennzeichnet, der sich in der Form einer veränderten FuE-Politik zeigte. Es formierten sich (2) Märkte in geschützten Nischen, in die (3) neue Unternehmen eintraten. Schließlich bildete sich (4) eine Art "Advocacy Coalition“ aus verschiedenen gesellschaftlichen Akteursgruppen, die sich für den Ausbau der erneuerbaren Energien engagierte. Diese Entwicklungen zeigten sich in der formativen Phase zwar erst in Ansätzen, bei noch intaktem soziotechnischem Regime; allerdings war die Entwicklung bereits auch im politischen System verankert. Dann, mit dem Erlass des Stromeinspeisungsgesetzes aus dem Jahr 1990, begann das vorhandene soziotechnische Regime der Energieversorgung in Deutschland Risse zu bekommen (Jacobsson \& Lauber 2006: 272), was eine Takeoff-Phase der erneuerbaren Energien, insbesondere der Windenergie, ermöglichte. Diese Take-off-Phase war begleitet durch eine sich allmählich herausbildende Vision eines machbaren Übergangs zu einer auf erneuerbaren Energien basierenden Energieversorgung. Mitte der 90er Jahre war die entsprechende „Advocacy Coalition“ bereits so stark, um Auseinandersetzungen über die Ausgestaltung zentraler Rahmenbedingungen zu gewinnen. Im Jahr 1998, mit dem Antritt der rot-grünen Regierung, wurden Mittel und Wege gefunden, um die Markträume für erneuerbare Energien zu schützen und auszubauen. Hierfür wurden neue Akteure, wie Anlagenhersteller und -betreiber aus dem Bereich der erneuerbaren Energien, aber auch Verbände und Vertreter traditioneller Akteursgruppen in Politiknetzwerke eingebunden (Jacobsson \& Lauber 2006: 272). Zum Abschluss ihrer Analysen aus dem Jahr 2006 vermuteten Jacobsson und Lauber, dass es schwierig werden könnte, angesichts etablierter und gut vernetzter Akteure des alten sozio-technischen Regimes und vor dem Hintergrund eines durch Liberalismus und die Privilegierung von Kurzzeitprofitabilität gekennzeichneten Systems die für den Umbau des Energieversorgungssystems erforderliche Förderpolitik entsprechend lange durchzuhalten
(Jacobsson \& Lauber 2006: 272 f.). Wie die Diskussionen der vergangenen Jahre gezeigt haben, lagen die beiden Autoren mit ihrer Vermutung richtig.

Aktuelle Forschungsarbeiten zur Transformation des deutschen Energieversorgungssystems gehen davon aus, dass sie in vier Phasen abläuft, wobei der Beginn etwa auf das Jahr 1990 datiert wird (Henning et al. 2014). Die erste Phase, bezeichnet als „Entwicklung erneuerbarer Energien", reicht diesen Arbeiten zufolge von 1990 bis etwa 2010. Sie ist durch die Entwicklung der erforderlichen Basistechnologien gekennzeichnet, durch wesentliche Kostenreduktionen sowie durch Markteinführungen und den Ausbau der erneuerbaren Energien, ohne signifikante Implikationen für das Gesamtsystem der Energieversorgung. Die Phase zwei, genannt "Systemintegration", beinhaltet die Aktivierung von Flexibilitäten bei residualer Stromerzeugung und -nutzung, die Etablierung von Kurzzeitspeichern sowie die Durchsetzung des Demand Side Managements. Sie umfasst einen geschätzten Zeitraum von 20 Jahren und reicht von 2010 bis 2030. Dieser Phase schließt sich die sogenannte Phase drei "Synthetische Brennstoffe“ an, die von 2030 bis etwa 2050 reicht. Sie ist gekennzeichnet durch signifikante negative Residuallasten und die Nutzung von Strom aus erneuerbaren Energien zur Erzeugung synthetischer Brennstoffe. Diese werden insbesondere im Bereich Mobilität Verwendung finden. Die vierte Phase der Transformation schließlich wird für den Zeitraum ab 2050 angenommen ("Erneuerbare Energien Import"). Sie beinhaltet die vollständige Verdrängung fossiler Ressourcen und den Import von erneuerbaren Energieträgern, z.B. aus sonnenreichen Regionen (Henning et al. 2014). Die gesamte Transformation, die nach Auffassung dieser Autoren über einen Zeitraum von weit über 60 Jahren reicht, wird begleitet von einer kontinuierlichen Erhöhung der Effizienz auf der Seite des Energieverbrauchs. Die Transformation wird auf alle Wandlungsketten und Verbrauchssektoren bezogen.

Schließt man sich diesem Verständnis an, so befindet sich der Transformationsprozess gegenwärtig am Anfang 
der Phase zwei („Systemintegration $^{\text {“) }}$ und ein langer und unsicherer Weg ist absehbar. Auch deshalb hat das BMBF die Fördermaßnahme „Umwelt- und gesellschaftsverträgliche Transformation des Energiesystems“ (2014-2017) ins Leben gerufen, die davon ausgeht, dass die Transformation des Energiesystems nicht technokratisch top-down oder gar im Detail gesteuert werden kann, sondern als gesellschaftlicher Such-, Verhandlungs- und Entscheidungsprozess konzipiert sein muss, der forschungs- und wissensbasiert ist (Institut für sozial-ökologische Forschung 2014). Dies führt unmittelbar zu der Frage nach der zukünftigen Ausgestaltung dieses Weges und methodisch zu dem Problem, wie diese Zukunft so antizipiert werden kann, dass sinnvolle Handlungsoptionen ableitbar sind.

Um den Transformationsansatz weiter zu operationalisieren, wird das sozio-technische Regime der Energieversorgung von den Autoren als Energieversorgungssystem beschrieben, das durch vertikale Wertschöpfungsstufen, Systemkomponenten und entsprechende Akteursgruppen charakterisiert ist (vgl. Abb. 1). Treiber der Transformation dieses Systems sind interne Faktoren, wie beispielsweise neue Energietechnologien und Akteure, die neue Technologieentwicklungen vorantreiben sowie externe Faktoren, die eher der soziotechnischen Landschaft zuzuordnen sind, wie etwa europäische Klimaschutzziele oder übergreifende politisch-rechtliche Rahmenbedingungen.

Zum Energieversorgungssystem gehören die einzelnen Wertschöpfungsstufen, von der Primärenergiegewinnung über den Energietransport, die Energiewandlung, die Energieverteilung und den Energiehandel bis hin zum Endenergieverbrauch. Diesen Wertschöpfungsstufen entsprechend kommen Komponenten des Systems hinzu, von Branchen der Primärenergiegewinnung über technische Infrastrukturen bis hin zu den verschiedenen Energienutzungsbereichen wie Industrie, Handel, Gewerbe und Dienstleistungen. Den Wertschöpfungsstufen und Systemkomponenten können entsprechende Akteursgruppen zugeordnet werden, von Unternehmen bis hin zu privaten Haushalten.

\section{Methodisches Vorgehen}

Vor dem skizzierten konzeptionellen Hintergrund wurde ein strukturierter Szenarioanalyseprozess konzipiert, in dessen Mittelpunkt die Transformation des Energieversorgungssystems stand. Hierbei wurde berücksichtigt, dass für die „Antizipation von Energiezukünften" seit Jahrzehnten weltweit sehr erfolgreich modellgestützte Szenarien als Methode angewendet werden. Im Mittelpunkt dieser modellgestützten Szenarien stehen technisch-ökonomische Parameter, wie etwa Ausbaupfade für erneuerbare Energien oder Energiemixe. Die quantitative Erfassung von Energieflüssen im komplexen System der Umwandlungs-, Verteilungs- und Nutzungsprozesse hat sich hierbei als deren Stärke erwiesen (Weimer-Jehle et al. 2013: 27). Wenig explizit werden in solchen Szenarien die gesellschaftlichen Kontextbedingungen für die Szenarien dargestellt. Das betrifft beispielsweise den Wertewandel in der Gesellschaft, demografische Entwicklungen, politische Konstellationen, ökonomische Abhängigkeiten oder auch den Stand der Technologieentwicklung. Diese Kontexte beeinflussen die Energiesys-
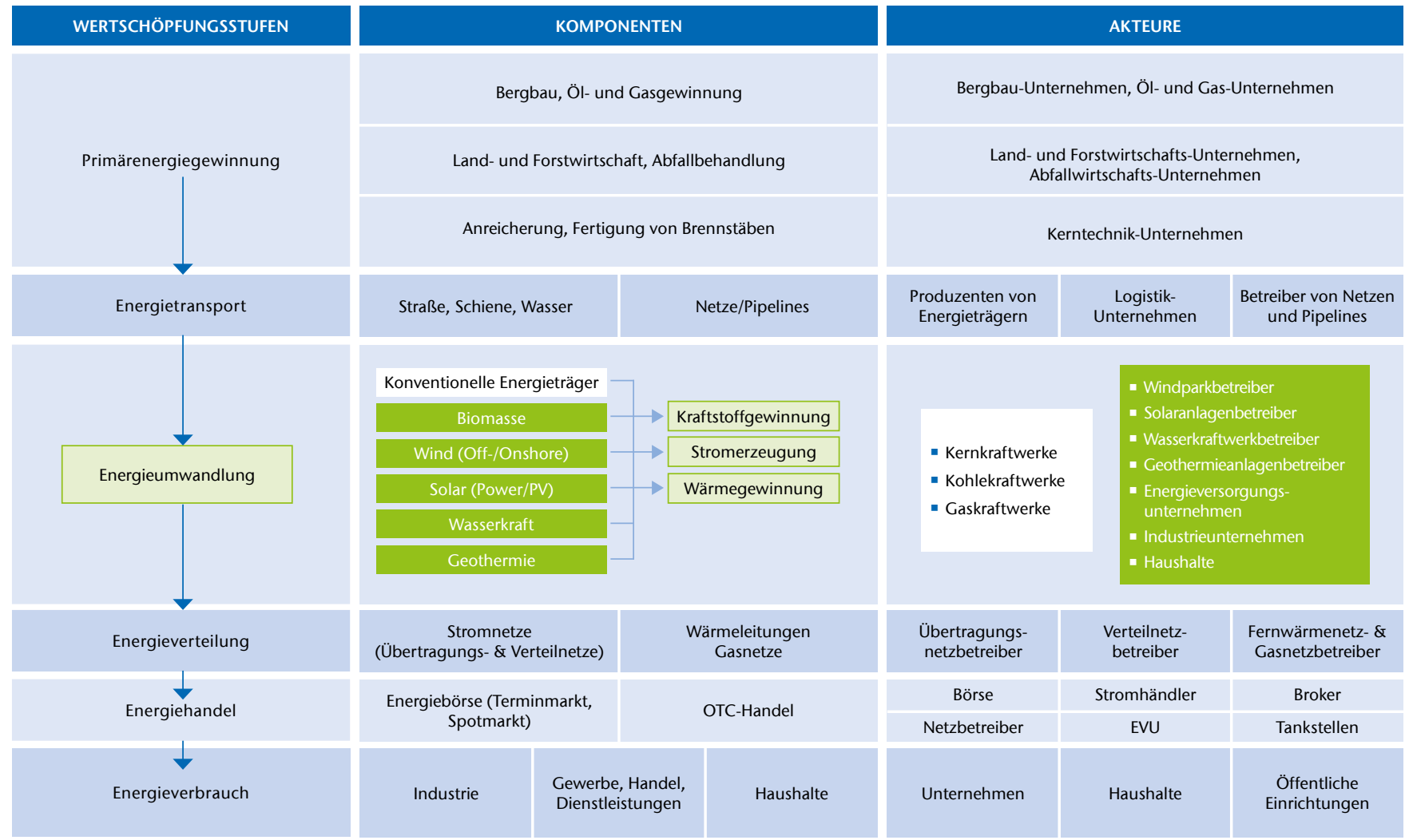
teme sehr stark und dauerhaft, sodass sie explizit zum Gegenstand der Szenarien gemacht werden sollten (Nielsen \& Karlsson 2007). Das Problem besteht hierbei in der systematischen Erfassung der Komplexität dieser gesellschaftlichen Kontextbedingungen sowie in den Unsicherheiten bei ihrer Projektion in die Zukunft.

Einen Lösungsansatz hierfür bilden hybride Szenarien, die einen qualitativen Kontextanteil und quantitativen „Kernanteil“ aufweisen. Die Erstellung der qualitativen Kontexte kann auf der Basis sogenannter Storylines (Gesellschaftsszenarien) erfolgen, für deren Entwicklung Expertenwissen aufbereitet wird. Weimer-Jehle et al. schlagen hierfür eine systematische Vorgehensweise vor und beziehen sich dabei auf die Methode der Cross-ImpactBilanzierung (CIB), die wiederum auf der Methode der Konsistenzanalyse basiert (Weimer-Jehle et al. 2013: 28). Sie zielt darauf ab, den systemanalytischen Gehalt der Kontextanalysen zu steigern. Im Verlauf der CIB-Methode werden zunächst die wichtigsten Faktoren identifiziert, die den Kontext beschreiben bzw. repräsentieren. Für diese werden dann alternative Entwicklungen repräsentiert und die Einflussbeziehungen zwischen den Faktoren systematisch herausgearbeitet. Im Ergebnis entsteht ein Wirkungsnetz dieser Faktoren, das den Kontext für modellbasierte Szenarien bilden kann (Weimer-Jehle et al. 2013: 31).

Im Unterschied hierzu werden für den Energiebereich auch sogenannte narrative Szenarien entwickelt, um intersubjektiv überprüfbare Zukunftsbilder zu entwerfen und die Kommunikation zwischen unterschiedlichen, an einem bestimmten Prozess beteiligten Gruppen zu unterstützen. Ihre Stärke besteht darin, dass sie nicht auf die Abbildung quantitativer Trends reduziert sind, sondern die Querbezüge zu den sozialen, wirtschaftlichen und technologischen Rahmenbedingungen sehr direkt herstellen (Venjakob 2012: 64).

Kronenberg et al. verglichen im Jahr 2012 sehr systematisch Energieszenarien für Deutschland (Kronenberg et al. 2012). Sie untersuchten 24 Szenariostudien, die im Zeitraum von 2007 bis 2011 erarbeitet wurden. Die Untersuchung wurde in der Form einer Stär-
ken-Schwächen-Analyse dargelegt, der Kriterien zugrunde lagen, um die Qualität der Szenarien zu bewerten. Auf einige dieser Kriterien wird im Folgenden kurz eingegangen, da sie bei der Konzipierung der eigenen Szenarioanalyse Berücksichtigung fanden (Kronenberg et al. 2012: $149 \mathrm{ff}$.).

1) Methodische Fundiertheit: Im Mittelpunkt der methodischen Qualität steht die Frage des Detaillierungsund Komplexitätsgrades bezogen auf das gesamte Energiesystem. So reicht beispielsweise die ausschließliche Betrachtung einzelner Sektoren des Energieversorgungssystems nicht aus, um daraus Rückschlüsse auf das Gesamtsystem ziehen zu können. Besondere Bedeutung wird von den Autoren der untersuchten Studien dem Strommarktsegment zugemessen (Kronenberg et al. 2012: 150).

2) Szenariobegründung/Rahmenszenarien: Als ein entscheidendes Kriterium für die Qualität von Szenarien wird deren Einbettung in den Kontext der Entwicklung des Energiesystems insgesamt und seiner Komponenten gesehen. Entsprechende Storylines sollten den Rahmen für quantitative Szenarien bilden. Wert wird auf die Verknüpfung von quantitativen und qualitativen Aspekten in den Szenarien gelegt.

3) Akteursbezüge: Dieses Kriterium bezieht sich auf den Einbezug von Annahmen über das zukünftige Verhalten von Akteuren, die für das Energiesystem relevant sind.

4) Maßnahmen: Die Autoren gehen von der Annahme aus, dass Aussagen in Szenarien an Bedeutung gewinnen, wenn sie mit technischen und politischen Maßnahmen kombiniert bzw. durch sie begründet werden. Vor allem die Tiefgründigkeit der Analyse solcher Maßnahmen gerät in den Blick der Bewertung der Qualität von Szenarien (Kronenberg et al. 2012: 160).

Das Fazit dieser Stärken-SchwächenAnalyse hat gezeigt, dass in den untersuchten Szenarien technischwirtschaftliche Faktoren eindeutig dominieren, politik- und sozialwissenschaftliche Erkenntnisse oder Methoden kaum oder gar nicht be- rücksichtigt werden. Auf der anderen Seite nehmen offenbar politikwissenschaftliche Foresight-Aktivitäten die technisch-wirtschaftlichen Szenarien nicht zur Kenntnis, sodass beide Forschungslinien in Deutschland unverbindlich nebeneinander stehen (Kronenberg et al. 2012: 162). Als besonders kritisch wird hervorgehoben, dass die Szenariobegründungen nicht ausreichend sind bzw. die Kontexte der Szenarien zu wenig berücksichtigt werden. Akteursbezüge sind mangelhaft ausgeprägt und die Rolle von Institutionen wird nicht systematisch reflektiert.

Diese Erkenntnisse berücksichtigend, haben sich die Autoren des vorliegenden Beitrages am klassischen Verständnis der Szenarioanalyse orientiert (vgl. z.B. Gausemeier et al. 1998: 3, Reibnitz 1991, Mietzner \& Reger 2005, Mietzner 2009, Reibnitz 2006). Demzufolge handelt es sich bei Szenarien um die Beschreibung komplexer, möglicher, zukünftiger Situationen, deren Eintreten nicht mit Sicherheit bestimmt werden kann sowie die Darstellung einer Entwicklung, die aus der Gegenwart zu dieser Situation führen kann. Hiermit unterscheidet sich die Szenarioanalyse von Prognosen. Ihr geht es nicht um das Fortschreiben von vergangenen und gegenwärtigen Entwicklungen einer bestimmten Situation, sondern um die Antizipation von möglichen, unterschiedlichen Zukünften. Die mit Szenarien entwickelten Zukunftsbilder sollen insbesondere den Ansprüchen genügen, konsistent, komplex und plausibel zu sein. Im Mittelpunkt der Szenarioanalyse stehen deshalb begründete Annahmen über die Entwicklung vernetzter Bedingungen und Einflussfaktoren. In der Literatur werden unterschiedliche Szenariotypen beschrieben. Für den nachfolgend dargestellten Szenarioanalyseprozess ist charakteristisch, dass explorative Szenarien entwickelt werden sollten. D. h. ausgehend von einer gut strukturierten Ausgangssituation sollten vorwärtsgewandt zukünftige Entwicklungspfade begründet werden. Darüber hinaus war beabsichtigt, deskriptive Szenarien zu entwickeln. Deskriptive Szenarien fokussieren auf Wirkungs-Zusammenhänge und beinhalten möglichst wenige Werturteile 
des Erstellers der Szenarien. Im Unterschied hierzu fließen in präskriptive Szenarien, die auf der Basis von Finalitätsbeziehungen erstellt werden, die Ziele des Anwenders der Szenarien ein.

Der Ablauf der Szenarioanalyse orientierte sich an einem Prozess, der sich aus fünf Phasen zusammensetzt. Er beginnt (1) mit der Strukturierung und Beschreibung des Szenariogegenstandes (Energieversorgungssystem). Dem schließt sich (2) die Identifikation von Einflussfaktoren der Transformation des Energieversorgungssystems an, wobei sowohl systeminterne als auch externe Einflussfaktoren identifiziert und analysiert werden. In diesem Arbeitsschritt werden Expertenworkshops und schriftliche Befragungen als Methoden eingesetzt. (3) Die Ermittlung von Schlüsselfaktoren basiert auf der softwaregestützten Vernetzung der Einflussfaktoren (ScenarioManager, ScMI 2013) und der Bestimmung des Charakters der Einflussfaktoren, je nach Stellung im vernetzten Systemzusammenhang. Es wird insbesondere Wert darauf gelegt, die Zusammenhänge zwischen den Faktoren inhaltlich zu beschreiben. In der Phase (4) werden auf einem mehrtägigen Szenarioworkshop in gemischten Teams Projektionen für die unterschiedlichen Sets von Schlüsselfaktoren für die Transformation des deutschen Energiesystems erarbeitet und diskutiert. Das Kernteam des Szenarioanalyseprozesses prüft daraufhin die Konsistenzen der erarbeiteten Projektionen, wobei wiederum das Softwaretool ScenarioManager von ScMI (ScMl 2013) zum Einsatz kommt. Im Ergebnis dieses Arbeitsschrittes können Vorschläge für die Bündelung von Szenarien erarbeitet und hierauf basierend (5) Szenarien beschrieben werden. In der Umsetzungsphase der Szenarioanalyse werden schließlich Anforderungen an Qualifikationen und Kompetenzen abgeleitet.

\section{Ergebnisse}

Im Ergebnis wurden für Deutschland die drei Szenarien "Deutschland auf dem schnellen Weg zur vollständigen Energiewende", "Deutschland auf dem steinigen Weg der Energiewende" und "Energiewende - Eingefrorener Traum vom Grün“ entwickelt. Sie basieren auf Projektionen der Schlüsselfaktoren (1) Nachfrage nach erneuerbaren Energien, (2) Förderpolitik, (3) Energieeffizienz, (4) Grad der Selbstversorgung, (5) Grad der Dezentralisierung, (6) Akzeptanz, (7) Entwicklung von Energiespeichern, (8) rechtliche Rahmenbedingungen und integrieren Inhalte aus der ausführlichen Analyse und Beschreibung des deutschen Energieversorgungssystems. Vergleicht man die drei Szenarien der Transformation des Energieversorgungssystems mit dem Ziel, zukünftige Anforderungen an Qualifikationen und Kompetenzen zu identifizieren, wird Folgendes deutlich: Die Wertschöpfungsstufen des Energieversorgungssystems sind, wie nicht anders zu erwarten, in differenzierter Art und Weise von der Energiewende betroffen. Auf der Wertschöpfungsstufe der Primärenergiegewinnung sieht sich insbesondere der Kohlebergbau durch den Transformationsprozess herausgefordert. Während er sich im Szenario 3 weitgehend stabilen Entwicklungsbedingungen gegenüber sieht, ist seine Entwicklung in den anderen beiden Szenarien rückläufig bzw. stark rückläufig. Hieraus resultieren zwar keine neuen Anforderungen an zukünftige Qualifikationen und Kompetenzen; diese Entwicklung ist jedoch unter arbeitsmarktlichen Gesichtspunkten relevant, da hierbei Arbeitsplätze verloren gehen bzw. andere Einsatzmöglichkeiten für Arbeitsplätze geschaffen werden müssen. Die Land- und Forstwirtschaft sowie die Abfallwirtschaft als Primärenergieerzeuger sind in allen drei Szenarien nur in geringem Maße von Veränderungen betroffen, neue Anforderungen an Qualifikationen werden in diesem Bereich nicht gesehen. Auf der Ebene des Transports der Primärenergie werden keine spürbaren Veränderungen im Zuge der Transformation des Energieversorgungssystems erwartet. Alle drei Szenarien gehen nicht explizit auf diese Wertschöpfungsstufe ein. Demzufolge zeichnen sich für entsprechende Akteursgruppen, wie beispielsweise Logistik-Provider oder Pipelinebetreiber, keine neuen Anforderungen an Qualifikationen ab.
Die Wertschöpfungsstufe Energieumwandlung umfasst neben der Umwandlung traditioneller Energieträger die Umwandlung regenerativer Energiequellen, wie beispielsweise Wasserkraft oder Windkraft, in Strom, Wärme und/oder Brennstoff/Treibstoff. Neben den Betreibern traditioneller Kraftwerke gehören als Akteure zu dieser Wertschöpfungsstufe insbesondere die Betreiber von Wind- und Solarkraftwerken, von Wasserkraftwerken und Geothermieanlagen sowie Industrieunternehmen mit eigenen Kraftwerkskapazitäten. Diese Akteure sind in den drei Szenarien in unterschiedlicher Weise von der Energiewende betroffen. Im Szenario 1 wird ein schneller Ausbau der erneuerbaren Energien beschrieben, was insbesondere die Wind- und Solarkraftwerke sowie die Biomasseanlagen in unterschiedlichen Konversionspfaden betrifft. Die entsprechenden Umwandlungsprozesse sowie die dazugehörenden Anlagen sind überwiegend bekannt. Dennoch werden zahlreiche Herausforderungen im Prozess der Energieumwandlung gesehen. Im Bereich der Bioenergie befinden sich beispielsweise Technologien wie die Holzstaubverfeuerung mit Motor/Turbine, die Holzvergasung im Zusammenhang mit der Brennstoffzelle oder auch die Biogasgewinnung und Brennstoffzelle noch in der Laborphase. Die Heißgasturbine, die Holzvergasung und Biokraftstoffsynthese oder auch Pyrolyse und Stromerzeugung hingegen befinden sich bereits in der Pilotphase (Eltrop et al. 2014: 8). Was die Umsetzung von Bioenergieprojekten betrifft, so hängen das Anspruchsniveau und demzufolge auch Anforderungen an Qualifikationen von der Komplexität und Verfügbarkeit typengeprüfter Anlagen bzw. Anlagenkomponenten ab, vom Umfang der Energieversorgung und vom erforderlichen Genehmigungsprozess. Während im ersten Szenario Bestrebungen hinsichtlich der Lösung des Problems der Abspaltung und Lagerung von Kraftwerks- $\mathrm{CO}_{2}$ als nicht sinnvoll erscheinen, passen sie sehr gut zum Szenario 3. Neue Qualifikationen und Kompetenzen könnten mit diesem Technologiepfad verbunden sein.

Das Szenario 1, mit einem verstärkten Einsatz von erneuerbaren Energien, 
geht weiterhin einher mit der Notwendigkeit der Entwicklung und des Einsatzes von Energiespeichern. Erste Modellprojekte mit großen Speicheranlagen (Power-to-Gas), modularen Batteriespeichern im Bereich $5 \mathrm{MW}$ und dezentralen Stromspeichern in privaten Haushalten deuten auf den Vormarsch von Speichertechnologien hin (vgl. FlZ Karlsruhe 2015). Während für die Errichtung und den Betrieb von großen Speicheranlagen nicht mit neuen Qualifikationsanforderungen in großem Umfang gerechnet wird, zeichnen sich für dezentrale Energiespeicher und deren Integration beispielsweise in Gebäude neue Anforderungen ab. Hierbei sind nicht nur Stromspeicher, sondern auch Wärmespeicher in der Diskussion.

Die Wertschöpfungsstufe der Energieverteilung, die Stromnetze sowie Gasund Wärmenetze umfasst, ist in erheblichem Maße von der Transformation des Energieversorgungssystems betroffen bzw. in diese involviert, was sich insbesondere im Szenario 1 mit einem hohen Grad von Dezentralität der Energieversorgung zeigt. Dies betrifft den Ausbau und die Optimierung der Übertragungsnetze, die in der Verantwortung der Übertragungsnetzbetreiber liegen, wobei davon ausgegangen wird, dass diese Unternehmen auch über die hierfür erforderlichen personalen Ressourcen verfügen. Auch bei den Verteilnetzen steht ein massiver Ausbau im Szenario 1 an, der verbunden sein wird mit einer zunehmend intelligenten Netzsteuerung. Mit der Energieflussüberwachung, die verbunden ist mit dem stärkeren Einsatz von Messtechnik, wird es möglich, neue Formen des Lastmanagements zu entwickeln und umzusetzen, was bedeutet, dass die unterschiedlichen Verbrauchergruppen durch zeitlich versetzte Nutzung überschüssiger Energie bzw. entsprechende Energieabgabe Kosten sparen bzw. Zusatzerlöse einnehmen können. Dies erfordert die weitere Entwicklung und den Einsatz von Smart-Grid-Technologien und -Modellen wie Smart Meter oder flexible Tarife (Strunz et al. 2012: 12). In diesem Bereich ist mit neuen Anforderungen an Qualifikationen sowohl bei der Planung, der Installation und dem Betrieb entsprechender Systeme zu rechnen.
Weitere Fortschritte in der Elektromobilität sind mit dem Ausbau der öffentlichen und privaten Ladeinfrastrukturen verbunden, die ebenfalls installiert und gewartet werden müssen, was zu spezifischen Anforderungen an die entsprechenden Betreiber führen kann. Im Unterschied hierzu wird davon ausgegangen, dass keine neuen Anforderungen für die Errichtung neuer Tankstelleninfrastrukturen (z. B. Wasserstoff) entstehen. Gleiches gilt auch für den Ausbau und den Betrieb von Gas- und Wärmenetzen im Zusammenhang mit der stärkeren Nutzung erneuerbarer Energien. Eine Ausnahme bilden hier die Schnittstellen zwischen den unterschiedlichen Netzarten. So kann beispielsweise durch dezentrale Blockheizkraftwerke eine geregelte Entnahme von Gas einhergehen mit der Wandlung in Strom und Wärme (Strunz et al. 2012: 13), was zu neuen Anforderungen an technische Kompetenzen führen kann.

Der Energiehandel wird in allen drei Szenarien vor neue Herausforderungen gestellt, da ein stärkerer Übergang zu marktwirtschaftlichen Regulierungsformen erfolgen wird. Betroffen werden Betreiber von ErneuerbareEnergie-Anlagen sein, Unternehmen, die die Direktvermarktung im Auftrag der Erzeuger übernehmen, Broker und Energieversorgungsunternehmen wie beispielsweise Stadtwerke. Aber auch große Unternehmen werden sich zukünftig in stärkerem Maße mit Beschaffungsstrategien für ihre Energieversorgung beschäftigen und entsprechende Qualifikationen nachfragen.

Auf der Wertschöpfungsstufe des Endenergieverbrauchs sind die privaten Haushalte, die Industrie, der Bereich Handel, Gewerbe und Dienstleistungen Akteure der Energiewende. Ihr Interesse besteht darin, einen bestimmten Nutzen mit minimalem Energieaufwand zu erreichen. Das betrifft Heizen, Kühlen und Warmwasser im Gebäude. Dieser Nutzen liegt insbesondere in den Themenfeldern Raumwärme, Warmwasser und Beleuchtung in Gebäuden, Prozessenergie in der Industrie sowie Kraft- und Treibstoffe im Verkehrssektor. Alle drei Szenarien gehen von einer wichtigen Rolle der Endenergieverbraucher bei der Transformation des Energieversorgungssystems aus. Vor allem im ersten
Szenario wird herausgearbeitet, dass der Primärenergieverbrauch erheblich sinken wird, wozu im Verkehrssektor die zunehmende Elektromobilität beitragen wird, mit neuen Anforderungen an die Ladeinfrastruktur, an Geschäftsmodelle von Flottenbetreibern sowie an Wartung und Reparatur. Im Bereich der Industrie wird mit der umfangreichen Einführung von Energiemanagementsystemen gerechnet, was mit neuen Anforderungen an geeignete Energiemanagementberater einhergehen wird. Die Effizienzsteigerung in Gebäuden ist ein sehr differenziertes Themenfeld, was sowohl Einfamilienhäuser als auch Mehrfamilienhäuser, Altbau und Neubau sowie Quartierseffizienz betrifft. Hier entstehen neue Anforderungen für das Handwerk, was die Systemintegrationsleistungen betrifft, für die Wohnungswirtschaft und Kommunen, um den Gebäudebestand sinnvoll zu sanieren.

\section{Schlussfolgerungen}

Unter methodischem Gesichtspunkt ergeben sich insbesondere zwei Schlussfolgerungen. (1) Die Aussagefähigkeit von Szenarien steigt, wenn Szenarioanalyseprozesse konzeptionell verortet werden, wie dies hier am Beispiel des Konzeptes „Multi-Level-Perspektive“ skizziert wurde. Eine erfolgreiche Operationalisierung setzt dabei voraus, dass sich Szenario-Teams nicht nur intensiv mit der Gegenwart ihres Gegenstandes beschäftigten, sondern stärker auch dessen Geschichte in den Blick nehmen, um ihre Analyse in übergreifende Prozesse einordnen zu können. Eine sehr systematische Beschreibung des Szenariogegenstandes (hier des Energieversorgungssystems) ermöglicht Szenarien, aus denen auch sehr konkrete Handlungsansätze im Prozess des Szenariotransfers mit vergleichsweise kurzen Zeithorizonten abgeleitet werden können. (2) Eine systematische Verknüpfung von quantitativen, modellbasierten Szenarien und eher qualitativ beschreibenden (narrativen) Szenarien erweist sich nach wie vor als eine anspruchsvolle methodische Herausforderung, die eine engere interdisziplinäre Zusammenarbeit, insbesondere von Natur- und Sozialwissenschaftlern, erfordert. 


\section{LITERATUR}

Eltrop L, Härdtlein M, Jenssen T, Özdemir ED, Henßler M Kruck C (2014) Grundlagen und Planung von Bioenergieprojekten. Dachleitfaden Bioenergie. Fachagentur Nachwachsende Rohstoffe, Gülzow-Prüzen. ISBN: 978 3-942147-13-2

FIZ Karlsruhe (2015) Energiespeicher - Forschung für die Energiewende. http://forschung-energiespeicher.info. Accessed 10 Aug 2015

Fournier J, Koske I, Wanner I, Zipperer V (2013) The Price of Oil - Will it Start Rising Again? OECD Economics Department Working Papers, vol 1031. OECD Publishing, Paris. doi: $10.1787 / 5 \mathrm{k} 49 \mathrm{q} 186 \mathrm{v} \times \mathrm{np}$-en

Gailing L, Hüesker F, Kern K, Röhring A (2013) Die räumliche Gestaltung der Energiewende zwischen Zentralität und Dezentralität. Explorative Anwendung einer Forschungsheuristik. Working Paper, vol 51. Leibniz-Institut für Regionalentwicklung und Strukturplanung, Erkner

Gausemeier J, Fink A, Schlake O (1998) Scenario Management: An Approach to Develop Future Potentials. Tech nol Forecast Soc Change 59(2):111-130. doi: 10.1016 S0040-1625(97)00166-2

Geels FW (2007) Transformations of Large Technical Systems. A Multilevel Analysis of the Dutch Highway System (1950-2000). Sci Technol Hum Val 32(2):123-14 $10.1177 / 0162243906293883$

Geels FW, Schot J (2007) Typology of sociotechnical transition pathways. Res Pol 36(3):399-417. doi: 10.1016/j. respol.2007.01.003

Grin J, Rotmans J, Schot J (2010) Transitions to Sustainable Development. New Directions in the Study of Long Term Transformative Change. Routledge, New York. ISBN: 978-0-415-87675-9

Henning HM, Palzer A, Schmidt M, Jachmann H, Strauß P, Pape C, Stock S, Fischedick M, Borggrefe F, Klann U (2014) Phasen der Transformation des Energiesystem - Ein ganzheitlicher Blick auf alle Wandlungsketten und Verbrauchssektoren. In: FVEE-Jahrestagung: Forschung für die Energiewende - Phasenübergänge aktiv gestalten, 6-7 Nov 2014, Berlin

Institut für sozial-ökologische Forschung (2014) Übergänge in eine nachhaltige Entwicklung. Wissenschaftliche Koordination der Fördermaßnahme Umwelt- und gesellschaftsverträgliche Transformation des Energie gesellschaftsverträgliche Transformation des Energie-
systems" des BMBF. http://www.isoe.de/projekte/aktuelle-projekte/transdisziplinaere-methoden-und-konzepte/wiko-soef-energie. Accessed 10 Sep 2015

Jacobsson S, Lauber V (2006) The politics and policy of energy system transformation-explaining the German diffusion of renewable energy technology. Energ Pol 34(3):256-276. doi: 10.1016/j.enpol.2004.08.029

Kronenberg $T$, Martinsen $D$, Pesch $T$, Sander $M$, Fische W, Hake JF, Kuckshinrichs W, Markewitz P (2012) Energieszenarien für Deutschland: Stand der Literatur und methodische Auswertung. In: Bruhns H (ed) Energiewende. Aspekte, Optionen, Herausforderungen. Deutsche Physikalische Gesellschaft, Bad Honnef, ISBN: 978 3-9811161-2-0, pp 132-166

Meadows DL, Meadows DH, Zahn E, Milling P, Heck H (1972) Die Grenzen des Wachstums. Bericht des Club of Rome zur Lage der Menschheit. DVA, Stuttgart. ISBN: 978-3-421-02633-0

Mietzner D (2009) Strategische Vorausschau und Szenarioanalysen. Methodenevaluation und neue Ansätze. Gabler, Wiesbaden. ISBN: 978-3-8349-1687-7. doi: 10.1007/978-3-8349-8382-4

Mietzner D, Reger G (2005) Advantages and disadvantages of scenario approaches for strategic foresight. Int I Tech Intell Plann 1(2):220-239. doi: 10.1504/IJTIP.2005.006516

Nielsen SK, Karlsson K (2007) Energy scenarios: a review of methods, uses and suggestions for improvement. Int J Global Energy Issues 27(3):302. doi: 10.1504/IJ GEI.2007.014350

Ohlhorst D, Tews K, Schreurs M (2013) Energiewende als Herausforderung der Koordination im Mehrebenensystem. TATuP 22(2):48-55

Reibnitz UH von (1991) Szenario-Technik. Instrumente für die unternehmerische und persönliche Erfolgsplanung. Gabler, Wiesbaden. ISBN: 978-3-409-13431-6
Reibnitz UH von (2006) From Anticipation to Action: How to put Foresight and Future Thinking into Concrete Action. Futures Res Q 22(2):5-14

ScMI (2013) Handbuch „Scenario-Manager“

Strunz K, Kuschke M, Schipper M, Viebig R (2012) Die Kompetenzen Berlin-Brandenburger Technologieunternehmen und Netzbetreiber für Energieversorgungssysteme. Szenarienanalyse - Übersicht regionaler Komsysteme. Szenarienanalyse - Übersicht regionaler Kom-
petenzen und Akteure. TSB Innovationsagentur Berlin petenzen und Akteure. TSB Innovations
$\mathrm{GmbH}$, Bereich Energietechnik, Berlin

Truffer B (2013) Zur geografischen Spezifizierung soziotechnischer Systeme. Energiewende zwischen lokaler Verankerung und globaler Vernetzung. TATuP 22(2):20-26

Venjakob I (2012) Qualitativ-narrative Szenarios für die langfristige Entwicklung des polnischen Energiesektors. Eine energiegeographische Untersuchung. Ecological Energy Policy, vol 13. ibidem, Stuttgart. ISBN: 978-38382-0354-6

WBGU (2011a) Transforming Energy Systems. Factsheet 2/2011. Wissenschaftlicher Beirat der Bundesregierung Globale Umweltveränderungen, Berlin. ISBN: 978-3936191-53-0

WBGU (2011b) Welt im Wandel. Gesellschaftsvertrag für eine große Transformation, 2nd edn. Wissenschaftlicher Beirat der Bundesregierung Globale Umweltveränderungen, Berlin. ISBN: 978-3-936191-38-7

Weimer-Jehle W, Prehofer S, Vögele S (2013) Kontextszenarien. Ein Konzept zur Behandlung von Kontextunsicherheit und Kontextkomplexität bei der Entwicklung von Energieszenarien. TATuP 22(2):27-36

\section{AUTOREN}

\section{Dr. phil. Frank Hartmann}

\section{Prof. Dr. rer. pol. Dana Mietzne}

Technische Hochschule Wildau

Forschungsgruppe Innovations- und Regionalforschung

E-Mail für Korrespondenz:

dana.mietzner@th-wildau.de 